\title{
A TOTALLY ORDERED INTEGRAL DOMAIN WITH A CONVEX LEFT IDEAL WHICH IS NOT AN IDEAL
}

\section{CHARLES HOLLAND}

The question of the existence of such a ring was raised by Donald Johnson. ${ }^{1}$ It suffices to find a cancellative totally ordered semigroup which has a lower segment $I$ that is a left ideal but not an ideal. For suppose that $S$ is such a semigroup and let $R$ be the semigroup ring of $S$ over the integers $Z$. Then $R$ can be ordered lexicographically (first by $S$ and then by $Z$ ) and it is well known that $R$ is an ordered integral domain. Let $g$ be the set of all elements of $R$ of the form $c_{1} x^{\gamma_{1}}+\cdots+c_{n} x^{\gamma_{n}}$, where $\gamma_{1}, \cdots, \gamma_{n} \in I$. Since $I$ is a lower segment of $S, g$ is a convex subgroup of $R$ and it is easy to verify that $g$ is a left ideal but not an ideal.

We now construct such a semigroup. The following ordered group, from which we shall extract $S$, is due to Conrad [1]. Let $H$ be the small direct sum of the integers over the integers ordered lexicographically. Define $\sigma$ from the integers to the ordered automorphism group of $H$ in the following manner: for $a \in H$ we use $a_{j}$ to denote the $j$ th component of $a$; then let $(a \sigma(i))_{j}=a_{j-i}$. Now let $G=Z \times H$ ordered lexicographically first by $Z$ then by $H$, where $(i, a)+(j, b)$ $=(i+j, a \sigma(j)+b)$. Then $G$ is an ordered group. Next let

$$
S=\left\{(i, a) \in G \mid i \leqq 0, a_{j} \geqq 0 \text { for all } j \text {, and } a_{j}=0 \text { for } j>0\right\} \text {. }
$$

It is easily seen that $S$ is a subsemigroup of $G$. Clearly $S$ is cancellative and contains the identity $(0,0)$ of $G$. Let

$$
I=\left\{(i, a) \in S \mid \text { either } i=-1 \text { and } a_{0}=0 \text {, or } i<-1\right\} .
$$

Then $I$ is a lower segment of $S$ which is a left ideal. But $I$ is not a right ideal because $(-1,0) \in I$ and if $(0, b) \in S$ such that $b_{0}>0$ then $(-1,0)$ $+(0, b)=(-1, b) \notin I$.

\section{REFERENCE}

1. P. Conrad, Extensions of ordered groups, Proc. Amer. Math. Soc. vol. 6 (1955) pp. 516-528.

\section{Tulane University}

Received by the editors December 5, 1959.

1 After this paper was submitted for publication the author learned that Johnson found a different solution to the problem, which will be published. 